\title{
Modelling of the processes of impact of a projectile with elements of individual defence
}

\author{
Aleksandr Kraus ${ }^{1,2}$, Evgeny Kraus, , , and Ivan Shabalin ${ }^{2}$ \\ ${ }^{1}$ Novosibirsk State Technical University, 630073, Novosibirsk, Russia \\ ${ }^{2}$ Khristianovich Institute of Theoretical and Applied Mechanics SB RAS, 630090, Novosibirsk, \\ Russia
}

\begin{abstract}
A two-dimensional and three-dimensional non-stationary problem of the interaction of a homogeneous impactor and a heterogeneous structure made of steel and ceramics and placed in a Kevlar pocket is considered. The model of the human body is a plate of gelatine with cylindrical inserts-imitators of human bones. The results of numerical simulation using different approaches for describing heterogeneous media are compared. On the basis of direct numerical simulation, it is shown that the gradient armor plate (steel $+\mathrm{B} 4 \mathrm{C})$ has the best weight and size parameters.
\end{abstract}

\section{Introduction}

Layered or heterogeneous armor plates are widely used for the protection of manpower and equipment [1]. Increase of strength characteristics and ballistic resistance of plates are caused by ballistic resistance of ceramics. The successful combination of acceptable strength characteristics and a reduction in weight, relative to a similar homogeneous plate, makes it necessary to study the interaction of impactors with such heterogeneous plates.

In [2], the reaction of ceramic and composite plates to high-speed impact was investigated. Using the finite element method, the mechanisms of perforation and energy dissipation in these plates were determined.

Optimization of double-layered armor subjected to a normal impact of $7.62 \times 54 \mathrm{~B} 32$ bullets was considered in [3]. Two cases of body armor were analyzed: when $\mathrm{Al}_{2} \mathrm{O}_{3}$ ceramics layer was supported by aluminum alloy AA2024-T3 and armor steel Armox 500T. As a result of the study, the panel optimization methodology was developed, which made it possible to determine the thickness of the panel layer with a minimum surface density.

Work [4] is devoted to the creation of an engineering method for describing the process of impact by an element on a two-layer barrier. In this article, analytical expressions are obtained for the maximum penetration rate into two-layer metal-ceramic and organoplastic ceramic targets. The structural characteristics and mechanical properties of the projectile and target materials are taken into account.

\footnotetext{
* Corresponding author: kraus@itam.nsc.ru
} 
The behavior of gelatin was described in [4-6]. The behavior of bone elements in a gelatin layer was considered in [7,8]. On basis of their simulation, the equation of state was formulated. Density, shear modulus, yield strength, etc. were obtained. Since human bones have a complex structure and significant anisotropy, the parameters in the paper were averaged over the transverse area of the bone. The speed of sound in the bones of a person has quite large variations depending on the direction of the impact, therefore, in [9], the average value of the speed of sound was chosen.

\section{The purpose of the work and formulation of the problem}

The purpose of this work is to investigate the behavior of protective element of body armor, to find acceptable weight and size parameters of the element made of heterogeneous compositions to reduce the impact effect on a person below the fatal characteristics.

The heterogeneous medium is a non-uniform system consisting of uniform parts separated by interface. The advantage of heterogeneous materials is that they have the same strength characteristics as homogeneous materials, but weight less.

Consider a two-dimensional axial non-stationary problem of the interaction of a uniform impactor with a protective element consisting of a plate placed in a Kevlar pocket. The core of a bullet of $7.62 \mathrm{~mm}$ caliber was chosen as the impactor, then the "core" The human body is imitated by a plate of gelatin with cylindrical inserts-imitators of human bones (Fig. 1).

Following problems [10-13], we use the model of solid deformable body, which consists of the equations of mass, momentum, and energy balance, as well as the equations of state and elastoplastic flow:

$$
\dot{\mathrm{x}}_{1}=\mathrm{u}_{\mathrm{i}}
$$

continuity equation

$$
\mathrm{V}_{0} \rho_{0}=\mathrm{V} \rho
$$

the law of material particle momentum variation

$$
\rho \dot{u}_{1}=\sigma_{i j, j} ;
$$

change in the particle internal energy

$$
\rho \dot{\mathrm{e}}=\frac{1}{2} \sigma_{\mathrm{ij}}\left(\mathrm{u}_{\mathrm{ij}}+\mathrm{u}_{\mathrm{ji}}\right) ;
$$

strain rate tensor has the form

$$
\dot{\varepsilon}_{1}=\frac{1}{2}\left(\mathrm{u}_{\mathrm{ij}}+\mathrm{u}_{\mathrm{ji}}\right)
$$

stress tensor we take in the generally accepted form

$$
\sigma_{i j}=-\delta_{i j} P+S_{i j},
$$

where $S_{i j}$ is the deviator of the stress tensor, which is responsible for the reaction to the shear form change of the material particle; $\delta_{\mathrm{ij}}$ is the Kronecker symbol; $\mathrm{P}$ is a function of pressure in the form of Mie - Gruneisen.

The process equations are taken in the Prandtl - Reuss form

$$
\widehat{\mathrm{s}}_{\mathrm{ij}}+\mathrm{d} \lambda^{\prime} \cdot \mathrm{s}_{\mathrm{ij}}=2 \mathrm{G} \dot{\varepsilon}_{\mathrm{ij}}^{\prime}, \dot{\varepsilon}_{\mathrm{ij}}^{\prime}=\dot{\varepsilon}_{\mathrm{ij}}-\dot{\varepsilon}_{\mathrm{kk}} / 3
$$

subject to the Huber - Mises plasticity

$$
s_{i j} \cdot s_{i j} \leq 2 Y_{0}^{2} / 3
$$


where $Y_{0}$ is the dynamic yield strength; scalar factor $d \lambda^{\prime}$ is determined using the wellknown procedure of reduction to the yield circle [10]. The above equations use the generally accepted notation: each of the indices $(i, j)$ runs through the values $1,2,3$; summation is performed over repeated indices; the point above symbol is the time derivative; the index after comma is the derivative with respect to the corresponding coordinate; $x_{i}, u_{i}$ are the components of the position and velocity vectors of material particle, respectively; $\rho$ is the current density; $G$ is the shear modulus.

The calculations were performed using software package "REACTOR" [14], where the numerical solution of the balance equations was implemented, taking into account the behavior of the materials and the realization of the boundary conditions.

\section{Models for construction of the heterogeneous material}

To determine the optimal parameters of body armor, a several models of building the heterogeneous environment were considered:

1. Layered environment, where each layer of armor plate has the properties and characteristics of specified homogeneous materials (Fig. 1);

2. The additive approach to the construction of material properties is a continuous material with a given mass concentration of the mixture components in the volume of the computational domain (Fig. 2) [15];

3. Direct numerical simulation of a heterogeneous material, which is a composition with given concentrations of the mixture components uniformly distributed throughout the computational domain (Fig. 3) [16,17];

4. Direct numerical simulation of a density-gradient material, which is a composition of a linearly distributed concentration of the mixture components within the computational domain (Fig. 4) [16,17].

Model 1: a protective design of two plates without gaps, made of steel and ceramics.

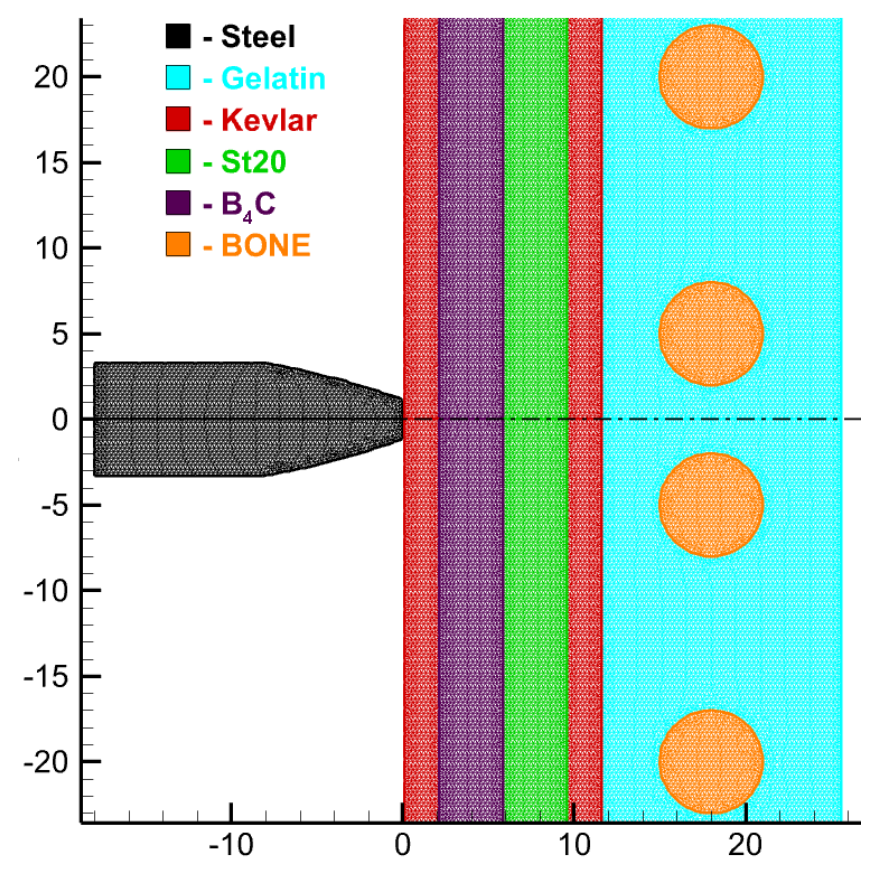

Fig. 1. The protective element is a two-layer plate (ceramic+steel) in a Kevlar pocket, model 1. 


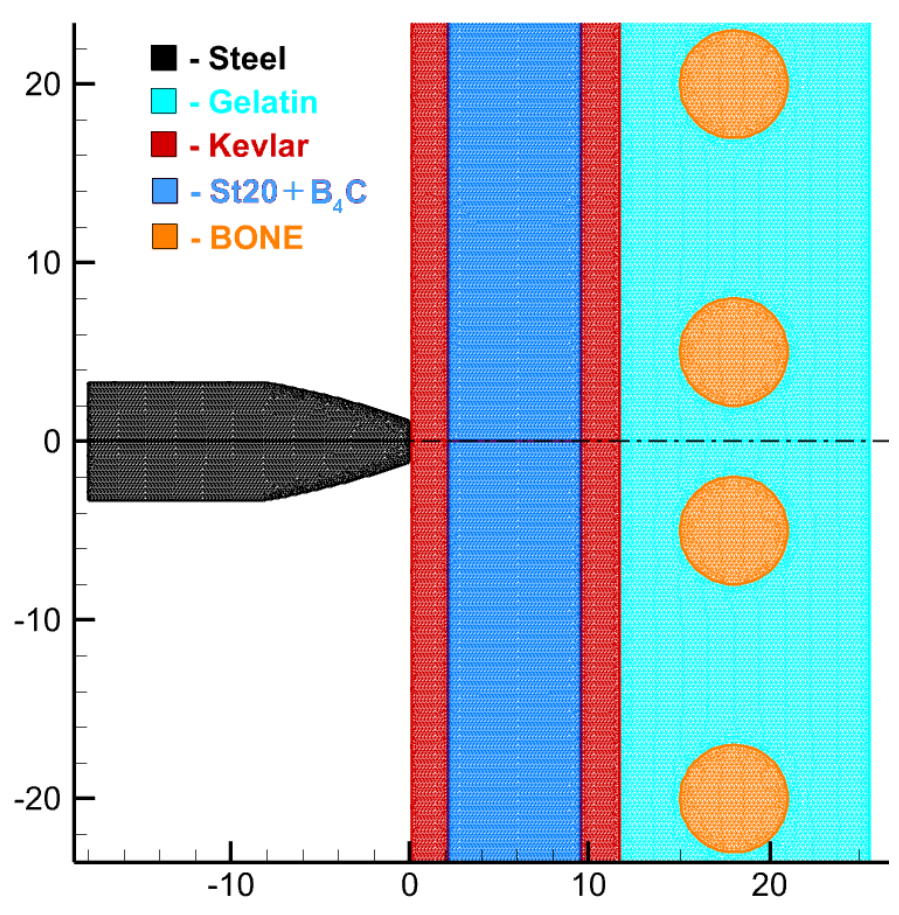

Fig. 2. Protective plate is the additive approximation of a mixture of ceramics and steel with effective environmental parameters, model 2.

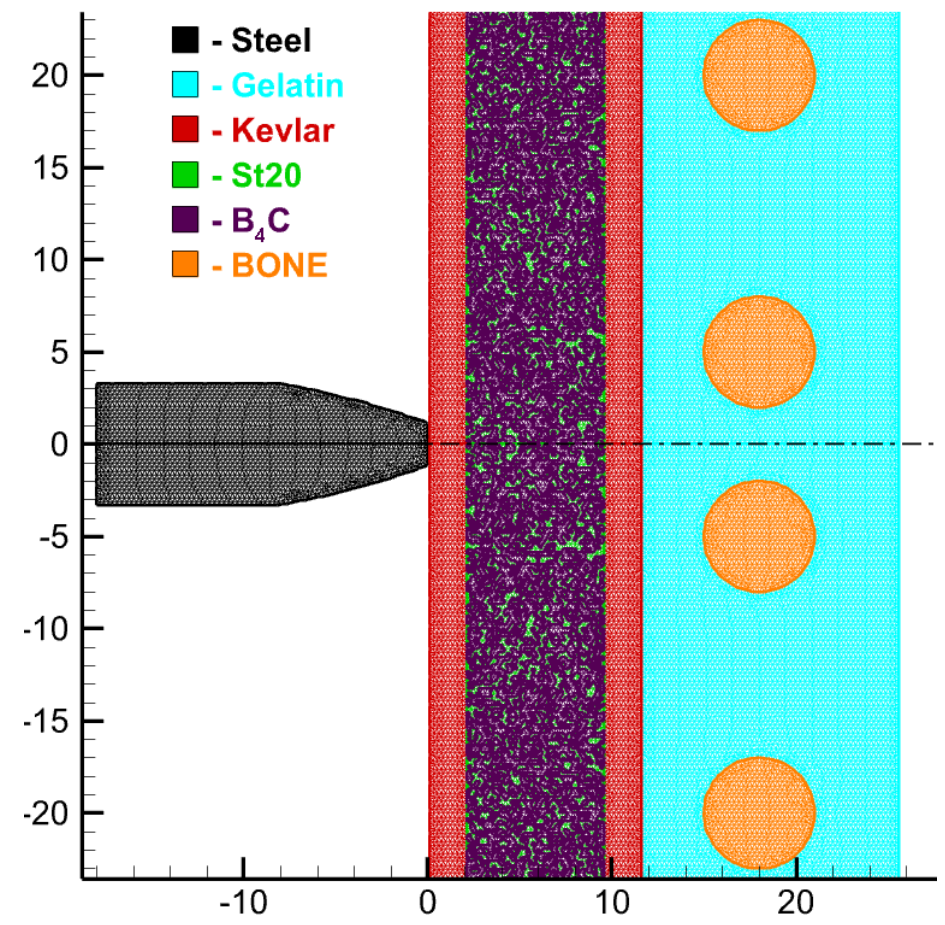

Fig. 3. The protective element with a plate of heterogeneous material obtained by the random distribution of ceramics in the steel volume with a given concentration, model 3. 
Model 2 is built using the additive approach. The material properties depend on the distribution function of the mass concentration $\alpha$. It creates an effective material with some averaged parameters based on the mass concentration [15].

The parameters are averaged according to [8]. Equation of state based on RankineHugoniot relations [18]

$$
\begin{gathered}
P=\frac{\rho_{0 \text { mix }} a_{\text {mix }}^{2}\left(1-\frac{\rho_{0 \text { mix }}}{\rho_{\text {mix }}}\right)}{\left[\left[1-\lambda_{\text {mix }}\left(1-\frac{\rho_{0 \text { mix }}}{\rho_{\text {mix }}}\right)\right]^{2}\right.}+P_{T} \\
a_{\text {mix }}=\left[\rho_{0 \text { mix }}\left(\sum \frac{\alpha_{i}}{\rho_{0 i}^{2} a_{i}^{2}}\right)^{1 / 2}\right]^{-1} \\
\lambda_{\text {mix }}=\left(1-\frac{\sum \alpha_{i} V_{0 i}\left[1-1 / \lambda_{i}\right]}{\sum \alpha_{i} V_{i}}\right)^{-1}
\end{gathered}
$$

$\mathrm{a}_{\mathrm{mix}}$ is the volumetric sound velocity in the mixture; $\lambda_{\text {mix }}$ is the slope of the generalized straight line in coordinates $\mathrm{D}_{\text {mix }}, \mathrm{U}_{\text {mix }}$.

Volume concentration

$$
\beta=\frac{\alpha \rho_{1}}{\sum \alpha \rho_{i}}
$$

Density

$$
\rho_{\text {mix }}=\left(\sum_{i=1}^{N} \frac{\alpha_{i}}{\rho_{i}}\right)^{-1}
$$

Shear modulus

$$
G_{\operatorname{mix}}=\left(\sum_{i=1}^{N} \alpha_{i} G_{i}\right)
$$

and yield strength

$$
\mathrm{Y}_{\text {mix }}=\left(\sum_{\mathrm{i}=1}^{\mathrm{N}} \beta_{\mathrm{i}} \mathrm{Y}_{\mathrm{i}}\right)
$$

Models 3 and 4 are direct numerical simulation of heterogeneous material. With this approach, particles of one material with their own characteristics are distributed over the volume of another material. Differences between models 3 and 4: the third one assumes the normal random distribution of the concentration of ceramics in the steel volume (Fig. 3), the fourth one assumes a gradient linear distribution of the ceramics concentration in the steel volume (Fig. 4). In both cases, the volume concentrations are the same. 


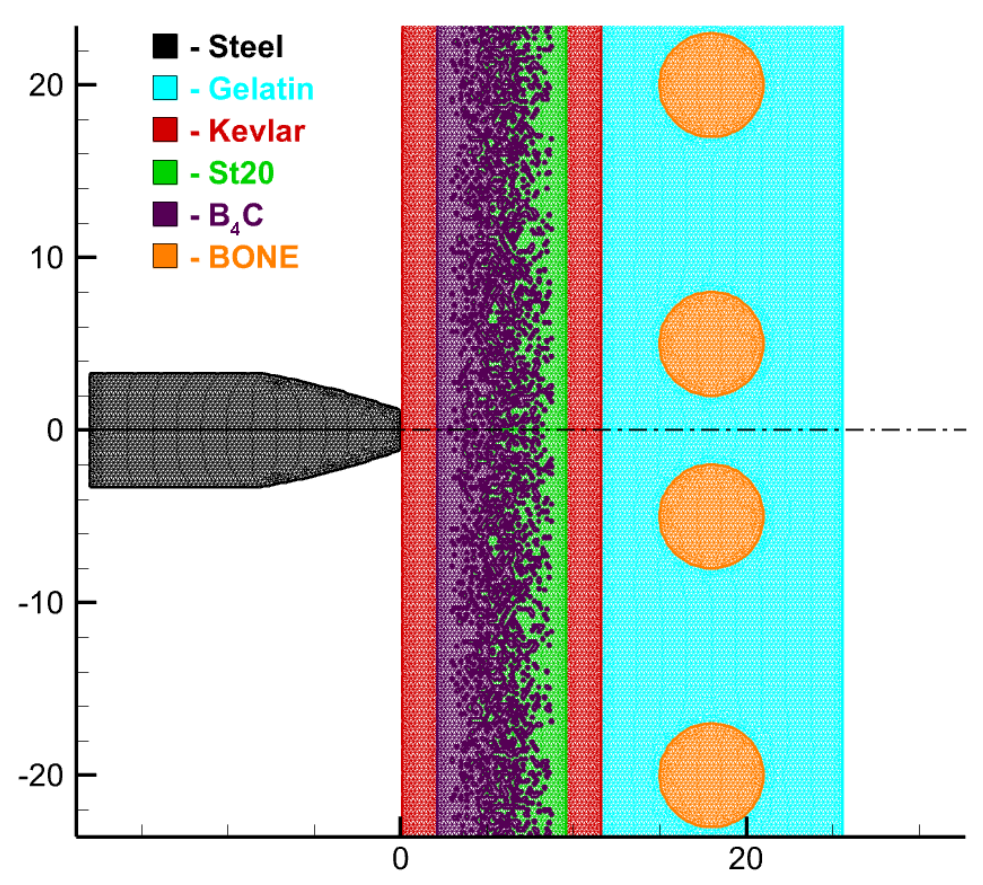

Fig. 4. The protective plate of a gradient material obtained by a linear distribution of the concentration of ceramics in steel, model 4.

\section{Simulation}

Since the configurations are defined, it is necessary to find the values of functions $\mathrm{u}, \mathrm{P}, \mathrm{V}, \mathrm{E}, \sigma_{\mathrm{i}}, \varepsilon_{\mathrm{i}}$ in the computational domains that satisfy the system of equations under the following conditions.

Initial conditions: at moment $\mathrm{t}=0$, all particles of the material are at rest and are evenly distributed. $\rho=\rho_{0} ; \mathrm{P}=0 ; \mathrm{S}_{\mathrm{i}}=0$. Initial velocity of the core $\mathrm{u}=400 \mathrm{~m} / \mathrm{s}$.

Boundary conditions: on the free boundary, $\bar{F}=(\sigma \bar{n})=0$; on the contact boundary, $\mathrm{F}^{+}=\mathrm{F}^{-}$and $\mathrm{u}_{\mathrm{n}}^{+}=\mathrm{u}_{\mathrm{n}}^{-}$, where $\mathrm{u}_{\mathrm{n}}$ is the velocity normal to the surface.

From the contact surface, both in the core and in the obstacle, a compression wave is formed with pressure amplitude of $\sim 1.5 \mathrm{GPa}$ (Fig. 5). Further, the compression wave propagates through the heterogeneous plate, and through the Kevlar layer it reaches the gelatin layer. The pressure values in the gelatin layer (at depth of $\sim 1 \mathrm{~cm}$ ) for all the models considered are presented in Table 1. It has been obtained that the pressure amplitude drops from 25 to 40 times when passing through the protective element, i.e. the parameters do not exceed the maximum permissible overpressure characteristics at the defeat of manpower [16]. 


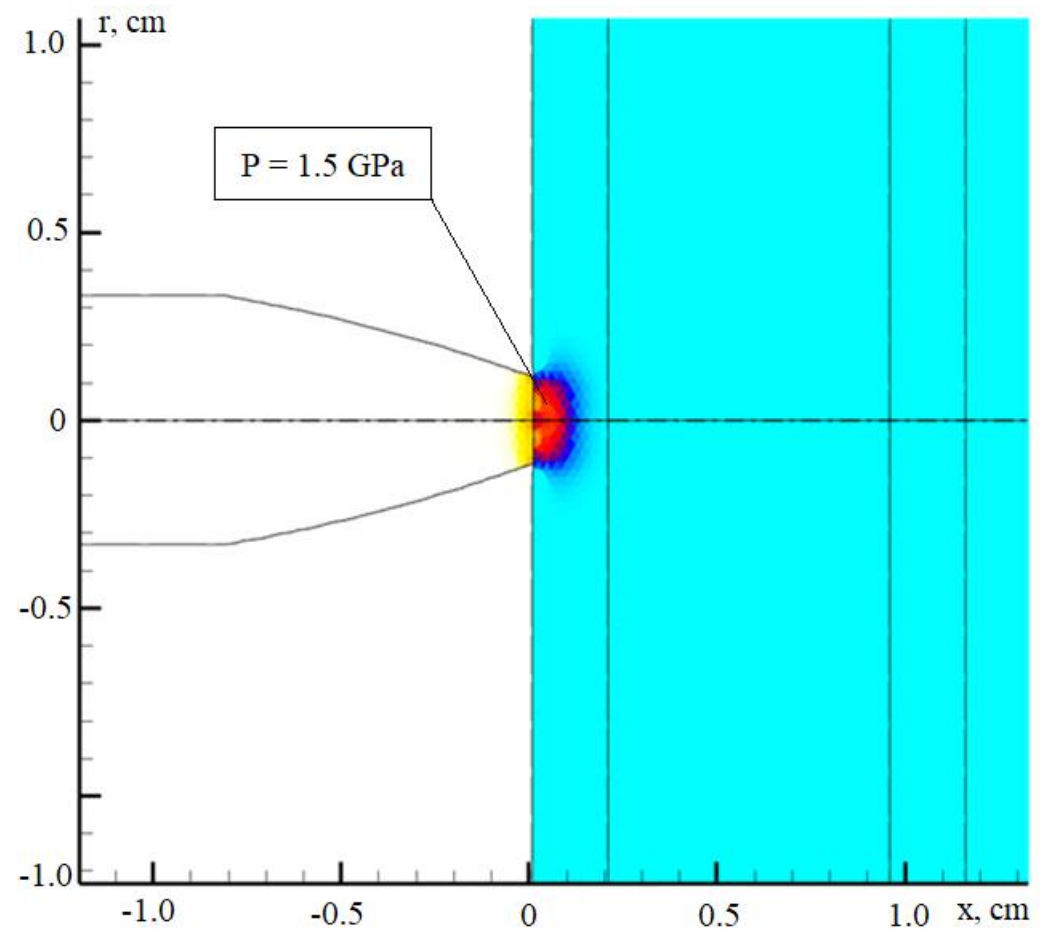

Fig. 5. The formation of a compression wave when the core contacts the protective element.

Table 1. The pressure values in the gelatin layer for all the models.

\begin{tabular}{|c|c|c|c|c|}
\hline & Model 1 & Model 2 & Model 3 & Model 4 \\
\hline $\mathrm{P}, \mathrm{MPa}$ & 70 & 44 & 40 & 48 \\
\hline
\end{tabular}

The core stops in the protective element, not punching it through at given initial speed of $400 \mathrm{~m} / \mathrm{s}$. In all cases, the outer layer of Kevlar is crushed by the incident core. The head part of the core acquires virtually the same shape for all combinations of the protective element. The differences are in the region of destruction of the front part of the plate.

The greatest pressure occurs in the two nearest bones in the direction of core movement, shown in Table 2. Due to the spherical shape, the wave attenuates and the pressure in the distant bones is less (Fig. 6).

Table 2. The greatest pressure occurs in the two nearest bones.

\begin{tabular}{|c|c|c|c|c|}
\hline & Model 1 & Model 2 & Model 3 & Model 4 \\
\hline $\mathrm{P}, \mathrm{MPa}$ & 4.6 & 6.43 & 5.67 & 3.47 \\
\hline
\end{tabular}




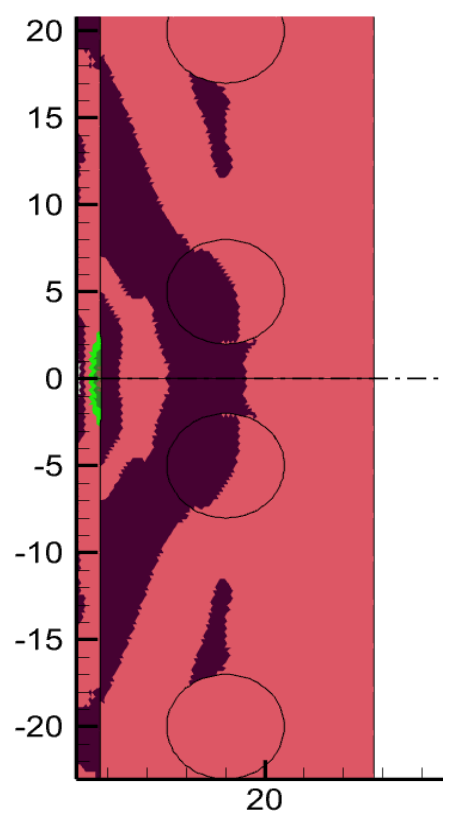

Fig. 6. Compression wave in the gelatin layer, $10 \mu \mathrm{s}$.

\section{Three-dimensional model}

To compare the results obtained, 3D geometric model was calculated. The comparison was performed with armored protection based on direct numerical simulation, since it showed the best results [17]. The human bone imitators were modeled with cylinders (Fig. 7). Geometric characteristics are similar to the two-dimensional problem, with difference in the thickness of the gelatin layer (Fig. 8).

For the three-dimensional model, the following results were obtained (see Table 3):

Table 3. The results for the three-dimensional model.

\begin{tabular}{|c|c|c|}
\hline Gelatin & Model 3 & Model 4 \\
\hline $\mathrm{P}, \mathrm{MPa}$ & 30.1 & 33.4 \\
\hline
\end{tabular}

\begin{tabular}{|c|c|c|}
\hline Bone & Model 3 & Model 4 \\
\hline $\mathrm{P}, \mathrm{MPa}$ & 2.9 & 2.86 \\
\hline
\end{tabular}

In 3D and 2D models, similar values are obtained. In the three-dimensional model, the parameters of the shock wave in the gelatin layer and in the bones do not exceed the permissible limit for humans. Also, the bones and the gelatin layer are not destroyed. 

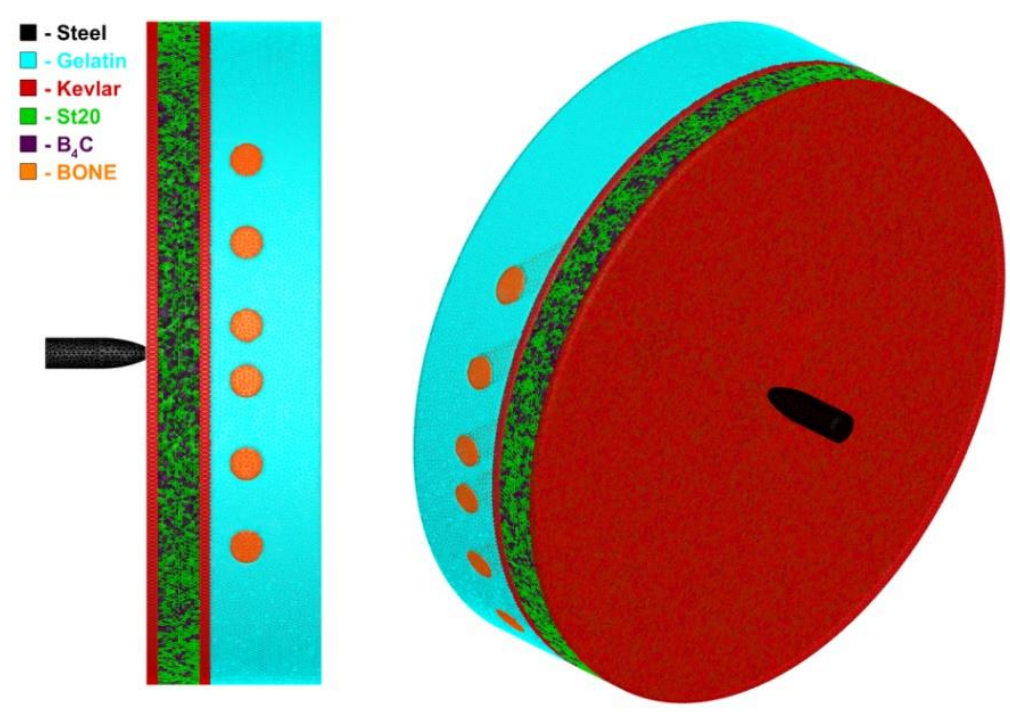

Fig. 7. Three-dimensional protective plate made of a gradient material obtained by randomly distributing the concentration of ceramics in steel (model 3).
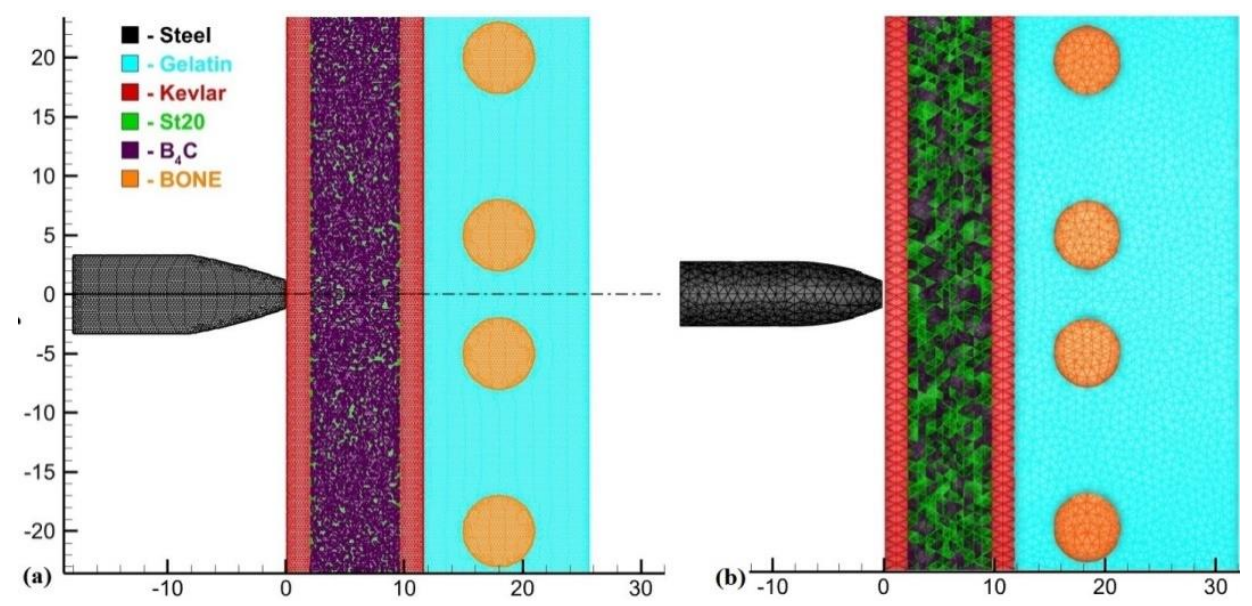

Fig. 8. Visual comparison of the two-dimensional (a) and three-dimensional models (b).

\section{Conclusions}

1. Despite the differences in the structure of the protective element, the output amplitudes of the velocity and pressure profiles are close at equal mass-dimensional parameters.

2. All protective elements withstand the impact of the core at meeting speed of $400 \mathrm{~m} / \mathrm{s}$. The pressure that comes to the imitator of the human body is less than the permissible limit.

3. The two-dimensional axial model and the three-dimensional model give similar results.

4. The most profitable in terms of mass and dimensional parameters is the model built on the principle of direct numerical simulation.

The work was supported by the Russian Foundation for Basic Research (project No. 19-08-00906). 


\section{References}

1. I.F. Kobylkin and V.V. Selivanov, Materials and Structures of Light Armor Protection (Bauman Moscow State Technical University, 2014)

2. A.A. Mobasseri, A.R. Ansari, H.R. Zarei, M. Sedighi, and F. Mobasseri, Aust. J. Basic Appl. Sci. 7, 6, 199 - 210 (2013)

3. P. Kędzierski, A. Morka, G. Sławiński, and T. Niezgoda, Bull. Polish Acad. Sci. Tech. Sci. 63, 1, 173 - 179 (2015)

4. S. S. Naarayan and G. Subhash, J. Mech. Behav. Biomed. Mater. 68, 32 - 41 (2017).

5. H. Frissane, L. Taddei, N. Lebaal, and S. Roth, Mech. Adv. Mater. Struct. 1-8 (2018)

6. Y. Huang, Determining the Equation of State Parameters for Ballistic Gelatin (2015)

7. J.R. Cameron, J.G. Skofronick, and R.M. Grant, Physics of the Body (Medical Physics Pub, 1999)

8. T.J. Horn, O.L.A. Harrysson, J.P. Little, H.A.W. Ii, D.J. Marcellin-Little, and E.P Fitts, in 21st Annual International Solid Freeform Fabrication Symposium-An Additive Manufacturing Conference, 864 - 875 (2010)

9. S. Pichardo, V. W. Sin, and K. Hynynen, Phys. Med. Biol. 56, 1, 219 - 250 (2011).

10. M.L. Wilkins, Computer Simulation of Dynamic Phenomena, Scientific Computation (Springer Berlin Heidelberg, 1999)

11. V.M. Fomin, A.I. Gulidov, G.A. Sapozhnikov, and I.I. Shabalin, High-Velocity Solids Interaction (SB RAS, 1999)

12. E.I. Kraus and I.I. Shabalin, AIP Conf. Proc. 2027, 030165 (2018)

13. A.V. Radchenko, P.A. Radchenko, and S.P. Batuev, Russ. Phys. J. 58, 3, 319 - 329 (2015)

14. E.I. Kraus and I.I. Shabalin, AIP Conf. Proc. 1770, 030092 (2016)

15. E.I. Kraus, V.M. Fomin, and I.I. Shabalin, PNRPU Mech. Bull. 9(1), 78-84 (2001)

16. E.I. Kraus, A.Y. Melnikov, V.M. Fomin, and I.I. Shabalin, J. Appl. Mech. Tech. Phys. 60, 3, 526 - 532 (2019)

17. A.E. Kraus and I.I. Shabalin, AIP Conf. Proc. 2027, 030166 (2018)

18. A.E. Kraus, E.I. Kraus, and I.I. Shabalin, AIP Conf. Proc. 2125, 030067 (2019) 